Review

\title{
Coenzyme Q10 and its Effective Sources
}

\author{
${ }^{1}$ Hamideh Vaghari, ${ }^{2}$ Roholah Vaghari, ${ }^{1}$ Hoda Jafarizadeh-Malmiri and ${ }^{3}$ Aydin Berenjian \\ ${ }^{I}$ Faculty of Chemical Engineering, Sahand University of Technology, Tabriz, Iran \\ ${ }^{2}$ Faculty of Nuclear Engineering, Shahid Beheshti University, Tehran, Iran \\ ${ }^{3}$ Faculty of Science and Engineering, School of Engineering, University of Waikato, Hamilton 3240, New Zealand
}

Article history

Received: 08-06-2016

Revised: 06-10-2016

Accepted: 07-10-2016

Corresponding Author:

Hamideh Vaghari

Faculty of Chemical

Engineering, Sahand University

of Technology, Tabriz, Iran

Email: vaghari_h@yahoo.com

\begin{abstract}
Coenzyme Q10 (2,3-dimethoxy, 5-methyl, 6-decaprenyl benzoquinone, CoQ10) is naturally present in many organisms. It has key roles in several biochemical pathways. CoQ10, as an electron and proton carrier for energy coupling leads to Adenosine Triphosphate (ATP) formation. Furthermore, in medicine, the pharmacological use of CoQ10 has attracted more attention due to its benefits in treating cardiovascular and degenerative neurologic diseases. CoQ10 can be produced by chemical synthesis, extraction from biological tissues and microbial fermentation. It is found in plants such as soya bean, peanut, palm oil and litchi pericarp and in animals such as pelagic fish, beef and pork hearts. Various analytical methods have been published for the extraction and analysis of CoQ10 from different matrices. Biological production of CoQ10 offers an environmentally benign option based on the enzymatic catalysis at the cellular level. Moreover, this process due to ease of control and low production costs offers more advantages over the existing technologies.
\end{abstract}

Keywords: CoQ10, Adenosine Triphosphate (ATP), Mitochondrial Enzymes, Extraction, Microbial Fermentation

\section{Introduction}

Coenzyme Q10 (2,3 dimethoxy, 5-methyl, 6decaprenyl benzoquinone, CoQ10) is present in many organisms (Fig. 1) (Xue et al., 2012). CoQ10 also known as ubiquinone or ubiquinone- 10 and its active form is ubiquinol, is abundant in plants, animals and microorganisms (Yuan et al., 2012). It plays a crucial role in the transfer of electrons between respiratory complexes of the electron transport chain, located within the inner mitochondrial membrane (Cluis et al., 2012).

Recently CoQ10 found a wide range of therapeutic applications (Tokdar et al., 2014; Langsjoen, 1994).

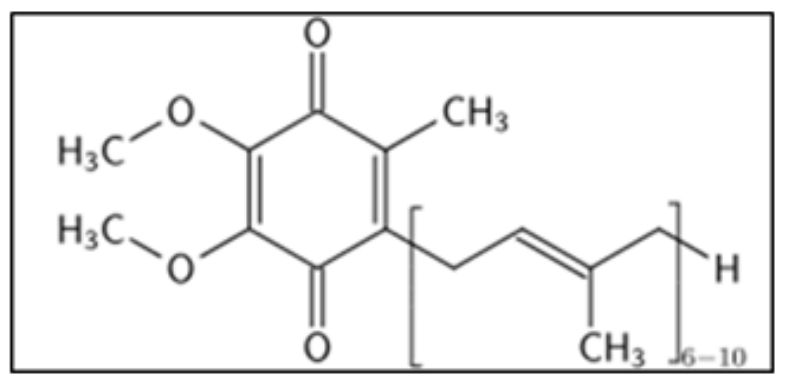

Fig. 1. Chemical structure of CoQ10 (Jankowski et al., 2016)
Extensive research has been conducted to increase CoQ10 production to meet growing demands for this product. CoQ10, can be produced by three methods: Chemical synthesis, extraction from biological tissues (animal and plant) and microbial fermentation (Laplante et al., 2009). Microbial biosynthesis offers several advantages over chemical synthesis and extraction including specificity towards the all-trans biologically active isomer of CoQ10 and the reduced production of environmentally hazardous waste based on the enzymatic catalysis at the cellular level for CoQ10 production (Cluis, 2012). Moreover, microbial fermentation found to be an attractive method for industrial production of CoQ10 (Lee et al., 2004; Park et al., 2005).

The present study aimed to discuss about importance, benefits of CoQ10 and also its effective sources and extraction methods.

\section{Importance and Benefits of CoQ10}

Application of CoQ10 in foods and animal tissue has attracted special attention owing to its crucial roles in many biochemical pathways (Rodriguez-Estrada et al., 2006). CoQ10 is the coenzyme for at least three mitochondrial enzymes (complexes I, II and III). 


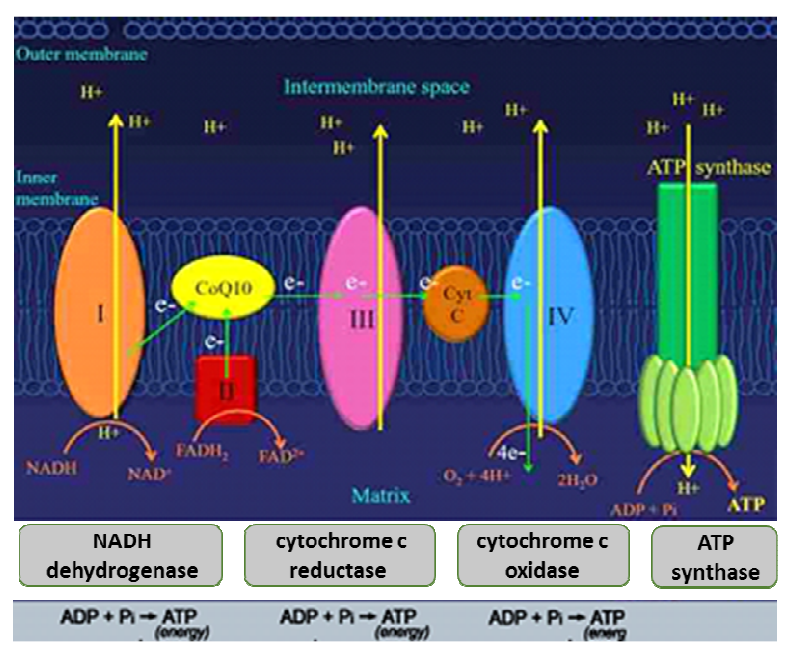

Fig. 2. Central role of CoQ10 in electron transport chain

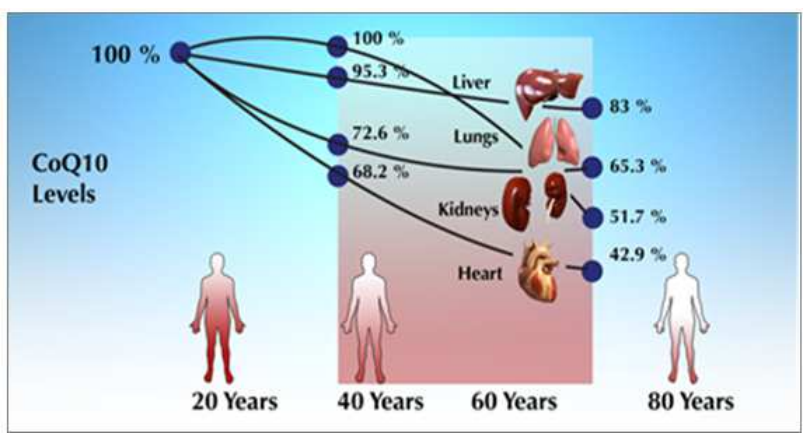

Fig. 3. CoQ10 decline with age (Littarru and Lambrechts, 2011)

CoQ10 as shown in Fig. 2 is a core component of cellular energy production. Due to its involvement in ATP synthesis, CoQ10 affects the function of every cell in the body, making it important for the health of all tissues and organs (de Dieu Ndikubwimana and Lee, 2014).

CoQ10 has been shown to have quite powerful antioxidant potential. Therefore, it can effectively defend against reactive oxygen species and free radical damage, protects the body from damage caused by harmful molecules (Ruiz-Jiménez et al., 2007) through protecting membranes and proteins from oxidation (Cluis, 2012). There is evidence that CoQ10 is playing a part in transcriptional regulation of genes, some of which play roles in inflammatory responses and in cholesterol metabolism (Schmelzer et al., 2007). Furthermore, in the medicine filed CoQ10 has received increasing attention due to its benefits in treating cardiovascular and degenerative neurologic diseases (Weant and Smith, 2005).

CoQ10 is naturally produced in the body, but its levels decrease as we age and may be low in people with cancer, genetic disorders, diabetes, heart problems and Parkinson's disease (Fig. 3). Symptoms of CoQ10 deficiency include heart failure, high blood pressure and chest pain. On the other hand, the concentration of CoQ10 in the body decreases year by year, indicating that it has a close relationship with aging (Fig. 2). For these reasons, some people rely on CoQ10 supplements. The daily intake of CoQ10 is suggested as $12 \mathrm{mg} \mathrm{kg}^{-1}$ (Rujiralai et al., 2014). More recently, nutraceutical supplements containing CoQ10 have gained a significant popularity in health management sections (Buettner et al., 2007).

Table 1. Overview of CoQ10 contents in various foods (Pravst et al., 2010)

\begin{tabular}{ll}
\hline Animal organ & $\mathrm{CoQ}_{10}$ concentration $[\mathrm{mg} / \mathrm{kg}]$ \\
\hline Beef & 113 \\
Heart & $39-50$ \\
Liver & $26-40$ \\
Muscle & $11.8-128.2$ \\
Pork & $22.7-54.0$ \\
Heart & $13.8-45.0$ \\
Liver & \\
Muscle & $116.2-132.2$ \\
Chicken & \\
Heart & $5-64$ \\
Fish & \\
Sardine & $43-67$ \\
Mackerel & $11-16$ \\
Red flesh & $4-8$ \\
White flesh & 5 \\
Salmon & \\
Tuna &
\end{tabular}

Table 2. Overview of CoQ10 contents in various plants (Pravst et al., 2010)

\begin{tabular}{ll}
\hline Plant & $\mathrm{CoQ}_{10}$ concentration $[\mathrm{mg} / \mathrm{kg}]$ \\
\hline Oils & $54-280$ \\
Soybean & $4-160$ \\
Olive & $64-73$ \\
Grapeseed & $4-15$ \\
Sunflower & 20 \\
Pistachio nuts & 17 \\
Hazelnuts & $5-14$ \\
Almond & \\
Nuts & 27 \\
Peanuts & 19 \\
Walnuts & $18-23$ \\
Sesame seeds & 20 \\
Pistachio nuts & 17 \\
Hazelnuts & $5-14$ \\
Almond & \\
Vegetables & $8-26$ \\
Parsley & $6-9$ \\
Broccoli & $2-7$ \\
Cauliflower & up to 10 \\
Spinach & $6-7$ \\
Grape & $2-5$ \\
Chinese cabbage & \\
Fruit & 10 \\
Avocado & 3 \\
Blackcurrant & 1 \\
Strawberry & $1-2$ \\
Orange & 1 \\
Grapefruit & 1 \\
Apple & \\
\hline & \\
& \\
&
\end{tabular}


CoQ10 supplements have shown positive effects on patients suffering from conjunctive heart failure and acute myocardial infarction (Hodgson et al., 2002; Yang et al., 2010). It has been proved that CoQ10 helps treat, muscular dystrophy and periodontal disease (Yang et al., 2010; Mancini and Balercia, 2011).

\section{CoQ10 Effective Sources}

CoQ10, can be produced by chemical synthesis, extraction from biological tissues (plants and animal) and microbial fermentation (Laplante et al., 2009). In the wake of environmental awareness, the chemical options became least desirable due to inherent uses of solvents and chemicals in the process (Tokdar et al., 2014).

\section{Plant and Animal Sources of CoQ10}

CoQ10 is naturally present in small amounts in a wide variety of foods, but is particularly high in animal meat organs such as heart, liver and kidney, beef as well as soy oil, sardines, mackerel and peanuts (Langsjoen,
1994). The highest content is found in meat and fish tissues and viscera due to their high levels of mitochondria (Reig et al., 2015). Moreover, presence of CoQ10 in bee pollen was investigated (Xue et al., 2012). The results of CoQ10 contents in animal organs and various plants are overviewed in Table 1 and 2 .

\section{Microbial Sources of CoQ10}

As summarized in Table 3, CoQ10 can be produced by microbial fermentation including fungi (e.g., Candida, Sporidobolus, Rhodotorula, Neurospora, Aspergillus) and bacteria (e.g., Agrobacterium, Paracoccus, Cryptococcusi, Rhodobacter, Tricosporon). Moreover, presence of CoQ10 in Artemia samples as a Crustacean was investigated (Rujiralai et al., 2014). Microbial production offers an environmentally benign option based on the enzymatic catalysis at the cellular level for CoQ10 assembly. Moreover, this approach is attractive to the industry because the process is easy to control at a relatively low production cost (Tokdar et al., 2014).

Table 3. CoQ10 production in wild types, chemical mutants and recombinant strains

\begin{tabular}{|c|c|c|}
\hline Source & Specific CoQ10 content (mg/g DCW) & Reference \\
\hline \multicolumn{3}{|l|}{ Wild type } \\
\hline Agrobacterium tumefaciens ATTC 4452 & 1.9 & Jeya et al. (2010) \\
\hline Agrobacterium tumefaciens KY-8593 & 1.2 & Cluis et al. (2007) \\
\hline Paracoccus denitrificans ATCC 19367 & 0.86 & Choi et al. (2005) \\
\hline Protaminobacter ruber & 1.52 & Jeya et al. (2010) \\
\hline Pseudomonas N84 & 1.2 & Jeya et al. (2010) \\
\hline Rhizobium radiobacter ATCC 4452 & 5.3 & Choi et al. (2005) \\
\hline Rhizobium radiobacter $\mathrm{A} 603-35$-gapA & 5.27 & Koo et al. (2010) \\
\hline Rhizobium radiobacter KCCM 10413 & 11.84 & Ha et al. (2009) \\
\hline Rhizobium radiobacter $\mathrm{T} 6102$ & 1.95 & Seo and Kim (2010) \\
\hline Rhizobium radiobacter WSH 2601 & 1.91 & Wu et al. (2003) \\
\hline Rhodobacter sphaeroides BCRC 13100 & 8 & Yen and Chiu (2007) \\
\hline Rhodobacter sphaeroides BCRC 13100 & 4.5 & Yen et al. (2010) \\
\hline Rhodobacter sphaeroides FERM-P4675 & 2.7 & Choi et al. (2005) \\
\hline Sporidiobolus johnsonii & 10.5 & Dixson et al. (2011) \\
\hline \multicolumn{3}{|l|}{ Recombinant strain } \\
\hline Escherichia coli & 0.29 & Choi et al. (2005) \\
\hline Escherichia coli & 1.41 & Choi et al. (2009) \\
\hline Escherichia coli & 2.428 & Zahiri et al. (2006) \\
\hline Escherichia coli & 0.44 & Huang et al. (2011) \\
\hline Escherichia coli & 0.45 & Huang et al. (2011) \\
\hline Escherichia coli & 3.24 & Huang et al. (2011) \\
\hline Escherichia coli & 0.51 & Zhang et al. (2007) \\
\hline Escherichia coli & 0.19 & Zhang et al. (2007) \\
\hline Escherichia coli & 0.77 & Zhang et al. (2007) \\
\hline Rhizobium radiobacter & 5.27 & Koo et al. (2010) \\
\hline Rhizobium radiobacter & 8.3 & Lee et al. (2007) \\
\hline \multicolumn{3}{|l|}{ Chemical mutants } \\
\hline Agrobacterium tumefaciens AU-55 & 9.6 & Choi et al. (2005) \\
\hline Agrobacterium sp. & 1.96 & Jeya et al. (2010) \\
\hline \multicolumn{3}{|l|}{ Agrobacterium tumefaciens } \\
\hline KCCM 10413 & 8.54 & Cluis et al. (2007) \\
\hline Agrobacterium tumefaciens KCCM 10413 & 9.71 & Jeya et al. $(2010)$ \\
\hline Rhodobacter sphaeroides & 8.7 & Jeya et al. $(2010)$ \\
\hline Rhodobacter sphaeroides Co-22-11 car & 2.6 & Cluis et al. (2007) \\
\hline Rhodobacter sphaeroides Co-22-11 & 2.5 & Choi et al. (2005) \\
\hline
\end{tabular}


However, due to the limits of CoQ10 accumulation in cells, strain improvements have been made using genetic engineering (using recombinant nucleic acid technology), chemical mutagenesis and high hydrostatic pressure treatment (Kim et al., 2015).

Industrial production of CoQ10 have predominantly relied on bacterial and yeast mutants due to their higher CoQ10 content (Tokdar et al., 2014). The isolation of strains by mutagenesis and selection on inhibitors has shown to be the most successful strategy to enhance CoQ10 yields (Yen and Shih, 2009). Table 2 summarizes CoQ10 production by some wild, chemical mutants and recombinant strains.

\section{CoQ10 Effective Extraction Methods}

Liquid-liquid extraction or ultrasound extraction by using a mixture of hexane and 2-propanol found to be the most common methods for extraction of CoQ10 from different samples (Xue et al., 2012). For example, CoQ10 from fresh tobacco leaves and litchi pericarp was extracted using ultrasonic extraction in the presence of ethanol and hexane (Rujiralai et al., 2014).

The two extraction methods generate a large amount of toxic chemicals within the process, which causes a significant environmental and health impact. Therefore, it is clearly preferable to obtain extracts by eliminating the use of toxic solvents (Xue et al., 2012).

Accelerated Solvent Extraction (ASE) was first developed by Dionex Corporation, in 1996 and then validated on a commercially-available, automated extraction system ASE a new extraction procedure for sample preparation, combines elevated temperatures and pressures with liquid solvents. Through this method organic solvents are used at high pressures and temperatures above the boiling point. In recent years, the popularity of ASE has increased since it can provide a higher extraction efficiency with low solvent volumes and a short extraction time in comparison with some classical extraction technologies such as liquid-liquid extraction and soxhlet extraction. ASE with ethanol and an acid- thermal treatment with a petroleum ether extractant were documented for extracting CoQ10 from bee-collected pollen and Agrobacterium tumefaciens, respectively (Richter et al., 1996).

\section{Conclusion Remarks}

CoQ10, a lipid-soluble endogenous pro-vitamin found naturally in the mitochondria, is present in many organisms. It has crucial roles in many biochemical pathways and important health functions. Levels of CoQ10 decrease as we age and may be low in people with cancer, genetic disorders, diabetes, heart problems and Parkinson's disease. For these reasons, some people rely on CoQ10 supplements.
CoQ10 can be produced from some microorganisms, plants and animals. It is important to establish a suitable extraction and analysis method for determining the content of CoQ10 in different foodstuffs. The most common methods for extracting CoQ10 from different samples are liquid-liquid extraction or ultrasound extraction. In recent years, the popularity of ASE has increased since it can provide higher extraction efficiency with low solvent volumes and a short extraction time in comparison with some classical extraction technologies. Microbial production offers an environmentally benign option and is attractive to the industry because of easy to control at a relatively low production cost. However the better precursors which could be combined for more CoQ10 production needs future studies. New methods for development of CoQ10 production in a better microorganism, which could produce high CoQ10 yield, could also be evaluated in the future. Finally, a type of reactor that provides high cell concentrations, high productivity and easy separation of the products could be determined from further research.

\section{Funding Information}

The authors have no support or funding to report.

\section{Author's Contributions}

All authors equally contributed in this work.

\section{Ethics}

This article is original and contains unpublished material. The corresponding author confirms that all of the other authors have read and approved the manuscript and no ethical issues involved.

\section{References}

Buettner, C., R.S. Phillips, R.B. Davis, P. Gardiner and M.A. Mittleman, 2007. Use of dietary supplements among United States adults with coronary artery disease and atherosclerotic risks. Am. J. Cardiol., 99: 661-666. DOI: 10.1016/j.amjcard.2006.09.116

Choi, J.H., Y.W. Ryu and J.H. Seo, 2005. Biotechnological production and applications of coenzyme $\mathrm{Q}_{10}$. Applied Microbiol. Biotechnol., 68: 9-15. DOI: 10.1007/s00253-005-1946-X

Choi, J.H., Y.W. Ryu, Y.C. Park and J.H. Seo, 2009. Synergistic effects of chromosomal ispB deletion and dxs overexpression on coenzyme $Q_{10}$ production in recombinant Escherichia coli expressing Agrobacterium tumefaciens dps gene. J. Biotechnol., 144: 64-69.

DOI: 10.1016/j.jbiotec.2009.04.010 
Cluis, C.P., A.M. Burja and V.J. Martin, 2007. Current prospects for the production of coenzyme $Q_{10}$ in microbes. Trends Biotechnol., 25: 514-521.

DOI: $10.1016 /$ j.tibtech.2007.08.008

Cluis, C.P., D. Pinel and V.J. Martin, 2012. The Production of Coenzyme Q10 in Microorganisms. In: Reprogramming Microbial Metabolic Pathways, Wang, X., J. Chen and P. Quinn, Springer Science and Business Media, ISBN-10: 9400750552, pp: 303-326.

de Dieu Ndikubwimana, J. and B.H. Lee, 2014. Enhanced production techniques, properties and uses of coenzyme $\mathrm{Q}_{10}$. Biotechnol. Lett., 36: 1917-1926. DOI: $10.1007 / \mathrm{s} 10529-014-1587-1$

Dixson, D.D., C.N. Boddy and R.P. Doyle, 2011. Reinvestigation of coenzyme Q10 isolation from Sporidiobolus johnsonii. Chem. Biodiversity, 8: 1033-1051. DOI: $10.1002 / \mathrm{cbdv} .201000278$

Ha, S.J., S.Y. Kim, J.H. Seo, M. Jeya and Y.W. Zhang et al., 2009. $\mathrm{Ca}^{2+}$ increases the specific coenzyme $\mathrm{Q}_{10}$ content in Agrobacterium tumefaciens. Bioprocess Biosyst. Eng., 32: 697-700. DOI: $10.1007 / \mathrm{s} 00449-009-0318-9$

Hodgson, J.M., G.F. Watts, D.A. Playford, V. Burke and K.D. Croft, 2002. Coenzyme $\mathrm{Q}_{10}$ improves blood pressure and glycaemic control: A controlled trial in subjects with type 2 diabetes. Eur. J. Clin. Nutrit., 56: 1137-1142. DOI: 10.1038/sj.ejcn. 1601464

Huang, M., W.A.N.G. Yue, L.I.U. Jianzhong and M.A.O. Zongwan, 2011. Multiple strategies for metabolic engineering of Escherichia coli for efficient production of coenzyme $Q_{10}$. Chinese J. Chem. Eng., 19: 316-326. DOI: $10.1016 /$ S1004-9541(11)60171-7

Jankowski, J., K. Korzeniowska, A. Cieślewicz and A. Jabłecka, 2016. Coenzyme Q10 - A new player in the treatment of heart failure? Pharmacol. Reports, 68: 1015-1019. DOI: 10.1016/j.pharep.2016.05.012

Jeya, M., H.J. Moon, J.L. Lee, I.W. Kim and J.K. Lee, 2010. Current state of coenzyme $Q_{10}$ production and its applications. Applied Microbiol. Biotechnol., 85: 1653-1663. DOI: 10.1007/s00253-009-2380-2

Kim, T.S., J.H. Yoo, S.Y. Kim, C.H. Pan and V.C. Kalia et al., 2015. Screening and characterization of an Agrobacterium tumefaciens mutant strain producing high level of coenzyme $\mathrm{Q}_{10}$. Process Biochem., 50: 33-39. DOI: $10.1016 /$ j.procbio.2014.10.024

Koo, B.S., Y.J. Gong, S.Y. Kim, C.W. Kim and H.C. Lee, 2010. Improvement of coenzyme $\mathrm{Q}_{10}$ production by increasing the NADH/NAD ${ }^{+}$Ratio in Agrobacterium tumefaciens. Bioscience, Biotechnol. Biochem., 74: 895-898. DOI: 10.1271/bbb.100034

Laplante, S., N. Souchet and P. Bry, 2009. Comparison of low-temperature processes for oil and coenzyme $\mathrm{Q}_{10}$ extraction from mackerel and herring. Eur. J. Lipid Sci. Technol., 111: 135-141.

DOI: $10.1002 /$ ejlt.200800133
Lee, J.K., D.K. Oh and S.Y. Kim, 2007. Cloning and characterization of the dxs gene, encoding 1-deoxyd-xylulose 5-phosphate synthase from Agrobacterium tumefaciens and its overexpression in Agrobacterium tumefaciens. J. Biotechnol., 128: 555-566. DOI: 10.1016/j.jbiotec.2006.11.009

Lee, J.K., G. Her, S.Y. Kim and J.H. Seo, 2004. Cloning and functional expression of the dps gene encoding decaprenyl diphosphate synthase from Agrobacterium tumefaciens. Biotechnol. Progress, 20: 51-56. DOI: 10.1021/bp034213e

Littarru, G.P. and P. Lambrechts, 2011. Coenzyme Q10: Multiple benefits in one ingredient. Oléagineux, Corps Gras Lipides, 18: 76-82.

DOI: 10.1051/ocl.2011.0374

Mancini, A. and G. Balercia, 2011. Coenzyme $\mathrm{Q}_{10}$ in male infertility: Physiopathology and therapy. Biofactors, 37: 374-380. DOI: 10.1002/biof.164

Park, Y.C., S.J. Kim, J.H. Choi, W.H. Lee and K.M. Park et al., 2005. Batch and fed-batch production of coenzyme $\mathrm{Q}_{10}$ in recombinant Escherichia coli containing the decaprenyl diphosphate synthase gene from Gluconobacter suboxydans. Applied Microbiol. Biotechnol., 67: 192-196. DOI: 10.1007/s00253-004-1743-y

Pravst, I., K. Žmitek and J. Žmitek, 2010. Coenzyme Q10 contents in foods and fortification strategies. Critical Rev. Food Sci. Nutrit., 50: 269-280. DOI: 10.1080/10408390902773037

Reig, M., M.C. Aristoy and F. Toldrá, 2015. Sources of variability in the analysis of meat nutrient coenzyme $\mathrm{Q}_{10}$ for food composition databases. Food Control, 48: 151-154. DOI: 10.1016/j.foodcont.2014.02.009

Richter, B.E., B.A. Jones, J.L. Ezzell, N.L. Porter and N. Avdalovic et al., 1996. Accelerated solvent extraction: $\square$ A technique for sample preparation. Analytical Chem., 68: 1033-1039. DOI: $10.1021 /$ ac9508199

Rodriguez-Estrada, M.T., A. Poerio, M. Mandrioli, G. Lercker and A. Trinchero et al., 2006. Determination of coenzyme $Q_{10}$ in functional and neoplastic human renal tissues. Analytical Biochem., 357: 150-152. DOI: 10.1016/j.ab.2006.06.013

Ruiz-Jiménez, J., F. Priego-Capote, J.M. Mata-Granados, J.M. Quesada and M.L. de Castro, 2007. Determination of the ubiquinol-10 and ubiquinone10 (coenzyme Q10) in human serum by liquid chromatography tandem mass spectrometry to evaluate the oxidative stress. J. Chromatography A, 1175: 242-248. DOI: 10.1016/j.chroma.2007.10.055

Rujiralai, T., R. Nirundorn, C. Wilairat, N. Heewasedtham and C. Chonlatee, 2014. Development of an effective extraction process for coenzyme $\mathrm{Q}_{10}$ from Artemia. Chem. Papers, 68: 1041-1048. DOI: 10.2478/s1 1696-014-0558-2 
Schmelzer, C., I. Lindner, C. Vock, K. Fujii and F. Doring, 2007. Functional connections and pathways of coenzyme Q10-inducible genes: An in-silico study. IUBMB Life, 59: 628-633.

DOI: $10.1080 / 15216540701545991$

Seo, M.J. and S.O. Kim, 2010. Effect of limited oxygen supply on coenzyme $\mathrm{Q}(10)$ production and its relation to limited electron transfer and oxidative stress in Rhizobium radiobacter T6102. J. Microbiol. Biotechnol., 20: 346-349. PMID: 20208439

Tokdar, P., P. Ranadive, R. Kshirsagar, S.S. Khora and S.K. Deshmukh, 2014. Influence of substrate feeding and process parameters on production of coenzyme Q10 using Paracoccus denitrificans ATCC 19367 mutant strain P-87. Adv. Biosci. Biotechnol., 5: 966-977. DOI: 10.4236/abb.2014.512110

Weant, K.A. and K.M. Smith, 2005. The role of coenzyme $\mathrm{Q}_{10}$ in heart failure. Ann. Pharmacotherapy, 39: 1522-1526. DOI: 10.1345/aph.1E554

Wu, Z., G. Du and J. Chen, 2003. Effects of dissolved oxygen concentration and DO-stat feeding strategy on $\mathrm{CoQ}_{10}$ production with Rhizobium radiobacter. World J. Microbiol. Biotechnol., 19: 925-928. DOI: 10.1023/B:WIBI.0000007322.19802.57

Xue, X., J. Zhao, L. Chen, J. Zhou, B. Yue and Y. Li et al., 2012. Analysis of coenzyme Q10 in bee pollen using online cleanup by accelerated solvent extraction and high performance liquid chromatography. Food Chem., 133: 573-578. DOI: 10.1016/j.foodchem.2011.12.085

Yang, X., G. Dai, G. Li and E.S. Yang, 2010. Coenzyme Q10 reduces $\beta$-amyloid plaque in an APP/PS1 transgenic mouse model of Alzheimer's disease. J. Molecular Neurosci., 41: 110-113.

DOI: $10.1007 / \mathrm{s} 12031-009-9297-1$
Yen, H.W. and C.H. Chiu, 2007. The influences of aerobic-dark and anaerobic-light cultivation on $\mathrm{CoQ}_{10}$ production by Rhodobacter sphaeroides in the submerged fermenter. Enzyme Microbial Technol., 41: 600-604.

DOI: $10.1016 /$ j.enzmictec.2007.05.005

Yen, H.W. and T.Y. Shih, 2009. Coenzyme $Q_{10}$ production by Rhodobacter sphaeroides in stirred tank and in airlift bioreactor. Bioprocess Biosyst. Eng., 32: 711-716. DOI: 10.1007/s00449-008-0294-5

Yen, H.W., C.Y. Feng and J.L. Kang, 2010. Cultivation of Rhodobacter sphaeroides in the stirred bioreactor with different feeding strategies for CoQ10 production. Applied Biochem. Biotechnol., 160: 1441-1449. DOI: 10.1007/s12010-009-8576-1

Yuan, Y., Y. Tian and T. Yue, 2012. Improvement of coenzyme Q10 production: Mutagenesis induced by high hydrostatic pressure treatment and optimization of fermentation conditions. BioMed Res. Int. DOI: $10.1155 / 2012 / 607329$

Zahiri, H.S., S.H. Yoon, J.D. Keasling, S.H. Lee and S.W. Kim et al., 2006. Coenzyme $\mathrm{Q}_{10}$ production in recombinant Escherichia coli strains engineered with a heterologous decaprenyl diphosphate synthase gene and foreign mevalonate pathway. Metabolic Eng., 8: 406-416. DOI: 10.1016/j.ymben.2006.05.002

Zhang, D., B. Shrestha, Z. Li and T. Tan, 2007. Ubiquinone-10 production using Agrobacterium tumefaciens dps gene in Escherichia coli by coexpression system. Molecular Biotechnol., 35: 1-14. DOI: 10.1385/MB:35:1:1 